\title{
A Framework for Assessing the Quality of Event Detection in Sensor Networks.
}

\author{
Shahram Mohrehkesh, Stephan Olariu and Michele C. Weigle \\ Department of Computer Science, Old Dominion University, Norfolk, VA, USA \\ \{smohrehk, olariu, mweigle\}@cs.odu.edu
}

\begin{abstract}
Despite the multitude of protocols that have been designed for event monitoring in sensor networks, very little work has been devoted to assessing the quality of these protocols in terms of their ability to provide timely and accurate information about events. The problem is challenging because of the combined effects of the stochastic nature of event arrival and duration and the stochastic nature of a sensor's sleepawake schedule. These various stochastic processes need to be modelled jointly in order to be able to reason, with any degree of confidence, about the quality of the event detection. The main contribution of this paper is a framework for assessing our confidence about the occurrence of events in sensor networks. Our framework models the interaction between the stochastic nature of an event arrival, the event duration, and the sensor sleep/awake schedule. By employing our framework, it is possible to configure individual sensor duty cycles to meet the requirements of mission-oriented applications in terms of timely and accurate information about events of interest.
\end{abstract}

\section{Categories and Subject Descriptors}

C.2.1 [Network Architecture and Design ]: Wireless communication; C.2 [COMPUTER-COMMUNICATION NETWORKS]: General

\section{Keywords}

Quality of Event Detection, Sensor Networks, Stochastic Events, Duty cycle, Sleep, Awake

\section{INTRODUCTION}

The past decade has produced numerous protocols for sensor networks $[9,8]$. Sensor networks must be able to reliably detect events of interest to the mission at hand. Event detection triggers human response, in accordance with mission

\footnotetext{
${ }^{*}$ Work supported by NSF grant CNS-1116238
}

Permission to make digital or hard copies of all or part of this work for personal or classroom use is granted without fee provided that copies are not made or distributed for profit or commercial advantage and that copies bear this notice and the full citation on the first page. Copyrights for components of this work owned by others than ACM must be honored. Abstracting with credit is permitted. To copy otherwise, or republish, to post on servers or to redistribute to lists, requires prior specific permission and/or a fee. Request permissions from permissions@ acm.org.

MiSeNet'13, October 4, 2013, Miami, Florida, USA

Copyright 2013 ACM 978-1-4503-2367-3/13/10 ...\$15.00.

http://dx.doi.org/10.1145/2509338.2509341 . semantics. To avoid wasting human resources, it is important that the likelihood of false alarms be minimized while reporting bona-fide events in a timely manner. Minimizing false alarms amounts to maximizing a sensor's certainty of the occurrence of an event. This is a challenging task because the event may be detectable for only a limited amount of time. For example, in a surveillance application, a sensor may detect the presence of an intruder for only a short time, and then the intruder may move to another location.

Our contribution is a framework for assessing the quality of event detection (QED, for short) given the probability distributions of event arrival, event duration and sensor sleep-awake times. In our framework, we define quality of detection in terms of event detection time, that is, the total amount of time during which the event was observed by the sensor. Our analysis reveals the impact on QED of various system parameters, including the attributes of the sleep-awake schedule and the duration and arrival distributions of events.

The paper is organized as follows. Section 2 introduces the sensor model as well as the event model that the sensors are tasked to observe. Section 3 models the observation time by each sensor. We also define formally the QED based on total observation time. Extensive simulation results, presented in Section 4 confirm our theoretical findings. A succinct review of relevant related work is offered in Section 5. Finally, Section 6 concludes the paper.

\section{THE SENSOR MODEL}

To promote network longevity, sensors alternate between sleep and awake periods $[1,11]$. In the sleep state, the sensors turn off their radio interfaces. During the awake period, the sensors are fully operational. The sleep-awake schedule can be fixed or can vary dynamically. Importantly, sensor synchronization is expensive and energy-consuming and, consequently, the sensors are assumed to be asynchronous. Recently, AbdelSalam and Olariu showed that despite sensors being asynchronous, there are important common stochastic properties of their behavior [1]. Sleeping sensors are inactive and their presence reduces the Effective Sensor Density, which is the density of active sensors. Designers need to balance energy saving with mission-specific requirements when dealing with sleep-awake scheduling.

The lengths of the awake and sleep periods of a generic sensor are random variables, denoted as $A$ and $S$, respectively. We note that $A$ and $S$ need neither be identically distributed nor independent. Refer to Figure 1 for an example. The convolution $C=A+S$ is the length of a whole 


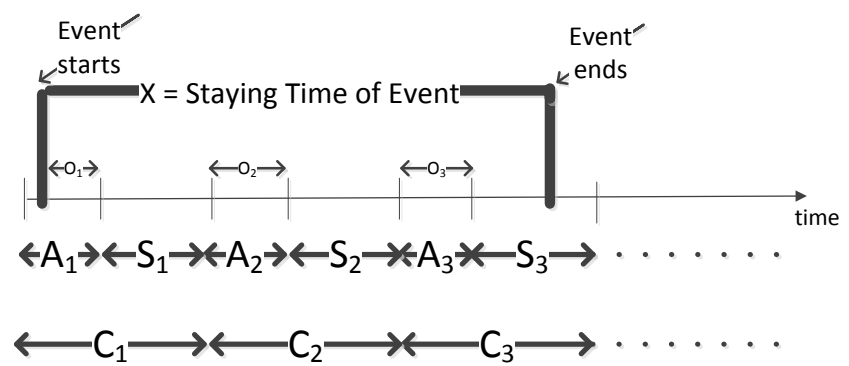

Total Observation Time $=\mathrm{O}_{1}+\mathrm{O}_{2}+\mathrm{O}_{3}$

Figure 1: Illustrating event occurrence.

cycle that is repeated over the entire lifetime of the sensor. Inspired by [1], we assume that $A$ and $S$ are random variables with finite expectation.

We define event observation time by a sensor as our metric for information collection about an event. Clearly, the longer an event is observed the more certain the sensor is about the occurrence of the event. We assume that a sensor is able to determine that multiple readings are caused by the same event, even when separated by several sleep periods. For example, when fire occurs, several high temperature readings can be attributed to the same fire event. For a given event, a sensor's observation time is the sum of all observations during successive awake periods. In Figure 1 , an event begins in awake period $A_{1}$ and ends in sleep period $S_{3}$. An event may arrive at a random point in time and has a random duration $X$, with finite expectation.

\section{OUR FRAMEWORK}

Given the stochastic nature of event arrival and duration in conjunction with the sleep-awake periods of sensors, we are interested in measuring the total observation time $O$ of an event by a generic sensor.

\subsection{Evaluating the total observation time}

Consider an event with random arrival time $L$ and duration $X$ with distribution functions $F_{L}$ and $F_{X}$, respectively. A sensor with random awake time $A$ and sleep time $S$ is tasked to detect the event. In order to model a realistic situation, we assume that all of these random variables have finite expectation. Write $C=A+S$ and let $O$ denote the total observation time of the event by our sensor.

Theorem 3.1. The conditional expectation $E[O]$ given that the event occurred at $t \in(0, C]$ is

$$
\begin{array}{r}
E[O]=\int_{0}^{A}\left(\int_{0}^{A-t}\left[1-F_{X}(x)\right] d x\right) d F_{L}(t)+ \\
\int_{0}^{C}\left(\sum_{i=1}^{\infty} \int_{i C-t}^{i C+A-t}\left[1-F_{X}(x)\right] d x\right) d F_{L}(t)
\end{array}
$$

There are four possible scenarios based on the time that the event begins and the time it ends. The event can occur when the sensor is awake or asleep and can end when the sensor is awake or asleep. For each scenario, we model the observation time. Then, the expected observation time is calculated based on the conditional expected value of each scenario.

\subsubsection{Event types}

Consider an arbitrary event that has occurred at $t \in(0, C]$ with a duration $X>0$. By the Law of Total Expectation, the (conditional) expected observation time $E[O]$ can be written as

$$
\begin{gathered}
E[O]=\int_{t=0}^{C} E[O \mid L=t] d F_{L}(t) \\
=\int_{t=0}^{A} E[O \mid L=t] d F_{L}(t)+\int_{t=A}^{C} E[O \mid L=t] d F_{L}(t)
\end{gathered}
$$

where two integrals in (3) correspond to the event occurring in $(0, A]$ and $(A, C]$, respectively. We classify events in terms of their ending time as follows.

Definition 3.2. An event is of Type I if

$$
t+X \in \bigcup_{j \geq 1}((j-1) C,(j-1) C+A)
$$

and of Type II if

$$
t+X \in \bigcup_{j \geq 1}((j-1) C+A, j C)
$$

Type I events terminate during a sensor awake period, while Type II events terminate when the sensor is asleep. As an illustration, Figure 1 features an event of Type II.

One can prove readily the following statements.

Claim 3.3. If $t \in(0, A]$ and the event is of Type I, then the corresponding total observation time is $X-(j-1) S$.

Claim 3.4. If $t \in(0, A]$ and the event of Type II, then the corresponding total observation time is $j A-t$.

Claim 3.5. If $t \in(A, C]$ and the event is of Type II, then the corresponding total observation time is $(j-1) A$.

Claim 3.6. If $t \in(A, C]$ and the event is of Type I, then the corresponding total observation time is $X+t-(j-1) S-A$.

\subsubsection{Events that begin during an awake period}

First, we consider events (of Type I and II) that begin when our sensor is awake, i.e. $t \in(0, A]$. To combine the observation time of Type I and Type II events, we define the $\operatorname{map} g:[0, \infty) \longrightarrow \mathbb{R}^{+}$as follows

$$
\begin{gathered}
g(X)= \\
\left\{\begin{array}{cc}
X-(j-1) S & \text { where } X \in(0, A-t) \cup \\
& \bigcup_{j \geq 1}((j-1) C-t,(j-1) C+A-t) \\
j A-t & \text { where } X \in \bigcup_{j \geq 1}((j-1) C+A-t, j C-t)
\end{array}\right.
\end{gathered}
$$

We show $\mathrm{g}(\mathrm{X})$ for small values of $j$ (number of sleepingawake cycles).

$$
g(X)=\left\{\begin{array}{cc}
X & 0 \leq X \leq A-t, j=1 \\
A-t & A-t<X \leq C-t, j=1 \\
X-S & C-t<X \leq C+A-t, j=2 \\
2 A-t & C+A-t<X \leq 2 C-t, j=2 \\
X-2 S & 2 C-t<X \leq 2 C+A-t, j=3 \\
3 A-t & 2 C+A-t<X \leq 3 C-t, j=3 \\
\text { etc. } &
\end{array}\right.
$$




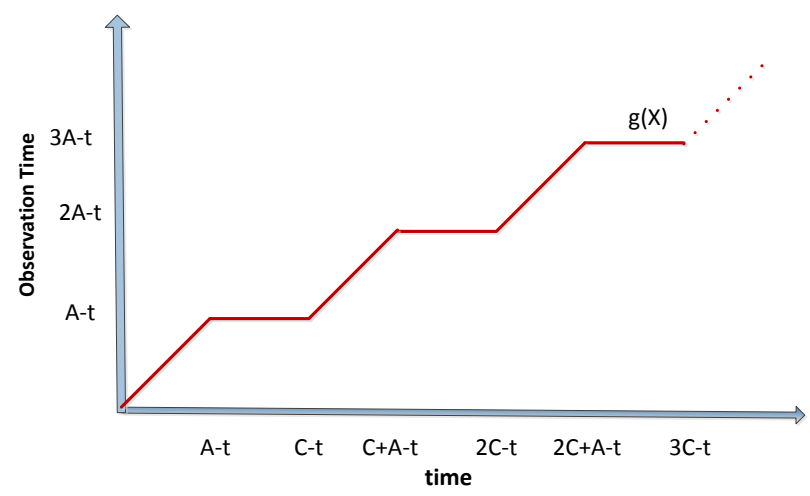

Figure 2: Observation time when the event begins during an awake period

The plot of $\mathrm{g}(\mathrm{X})$ is given in Figure 2 where we plot clock time on the horizontal axis and observation time on the vertical axis. By claims 3.3 and $3.4, \mathrm{~g}(\mathrm{X})$ is a continuous function. It is observed that for $t \in(0, A]$

$$
E[O \mid L=t]=E[g(X)]=\int_{X=0}^{\infty} g(X) d F_{X}(x)
$$

Note that since $X$ is a random variable, $g(X)$ is a random variable as well. Consequently, the first integral in the expression of $E[O]$ on (3) becomes

$$
\int_{t=0}^{A} E[O \mid L=t] d F_{L}(t)=\int_{t=0}^{A} \int_{X=0}^{\infty} g(X) d F_{X}(x) d F_{L}(t)
$$

\subsubsection{Events that begin during a sleep period}

Now, we consider events (of Type I and II) that begin when our sensor is asleep, i.e. $t \in(A, C]$. To combine the observation times of events of type I and II, we define the map $h:[0, \infty) \longrightarrow \mathbb{R}^{+}$as follows

$$
\begin{gathered}
h(X)= \\
\left\{\begin{array}{cc}
X+t-(j-1) S-A & \begin{array}{c}
\text { where } \\
X \in \bigcup_{j \geq 1}((j-1) C-t, \\
(j-1) C+A-t)
\end{array} \\
(j-1) A & \text { where } \\
& X \in \bigcup_{j \geq 1}((j-1) C+A-t, j C-t)
\end{array}\right.
\end{gathered}
$$

We show $\mathrm{h}(\mathrm{X})$ for small values of $j$ (number of sleepingawake cycles).

$$
h(X)=\left\{\begin{array}{cc}
0 & 0 \leq X \leq C-t, j=1 \\
X+t-C & C-t<X \leq C+A-t, j=2 \\
A & C+A-t<X \leq 2 C-t, j=2 \\
X+t-2 C+A & 2 C-t<X \leq 2 C+A-t, j=3 \\
2 A & 2 C+A-t<X \leq 3 C-t, j=3 \\
X+t-3 C+2 A & 3 C-t<X \leq 3 C+A-t, j=4 \\
3 A & 3 C+A-t<X \leq 4 C-t, j=4 \\
\text { etc. } &
\end{array}\right.
$$

The plot of $\mathrm{h}(\mathrm{X})$ is given in Figure 3. $h(X)$ is very similar to the plot $g(X)$. In fact, $h(X)$ can be seen as version of $g(X)$ shifted in time. It is now clear that for $t \in(A, C]$

$$
E[O \mid L=t]=E[h(X)]=\int_{X=0}^{\infty} h(X) d F_{X}(x) .
$$

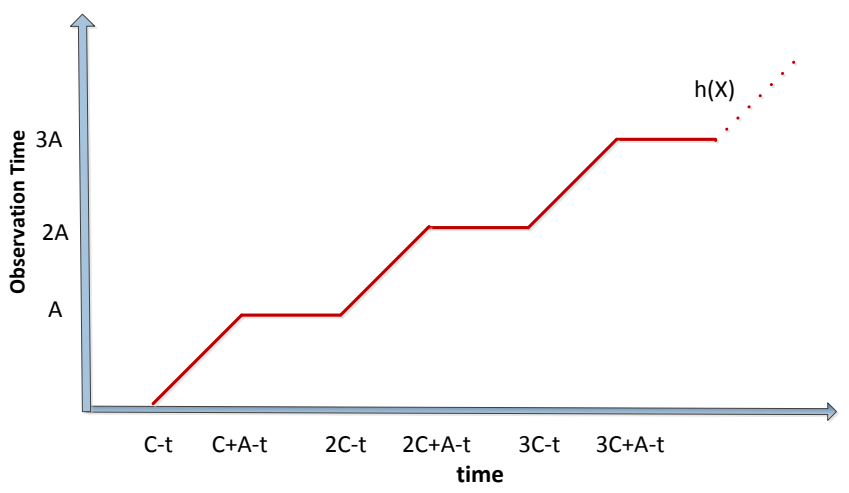

Figure 3: Observation time when the event begins during a sleep period

Consequently, the second integral in (3) becomes

$$
\int_{t=A}^{C} E[O \mid L=t] d F_{L}(t)=\int_{t=A}^{C} \int_{X=0}^{\infty} h(X) d F_{X}(x) d F_{L}(t)
$$

Finally the conditional expected observation time of the event is

$$
\begin{aligned}
E[O]= & \int_{t=0}^{A} \int_{X=0}^{\infty} g(X) d F_{X}(x) d F_{L}(t)+ \\
& \int_{t=A}^{C} \int_{X=0}^{\infty} h(X) d F_{X}(x) d F_{L}(t)
\end{aligned}
$$

In order to get a better handle on (10), we now look at the inner integrals.

\subsubsection{Expected observation for $g(X)$ and $h(X)$}

The purpose of this section is to find the expected observation time for each of $g(X)$ and $h(X)$. Proofs of following theorems can be found in Appendix B.

Theorem 3.7. If $\forall j \geq 1$ and assuming that $X$ has finite expectation,

$$
\begin{aligned}
& \int_{0}^{j C-t} g(X) d F_{X}(x)=\int_{0}^{j C-t} X d F_{X}(x)- \\
& \sum_{i=1}^{j} \int_{(i-1) C+A-t}^{i C-t}\left[1-F_{X}(x)\right] d x+j S\left[1-F_{X}(j C-t)\right]
\end{aligned}
$$

Next, since the limit of the integral is required in (10), Theorem 3.7 can be evaluated when $j$ goes to $\infty$. The results are captured by the following theorem.

Theorem 3.8.

$$
\begin{aligned}
& \int_{0}^{\infty} g(X) d F_{X}(x) \\
& =\int_{0}^{A-t}\left[1-F_{X}(x)\right] d x+\sum_{i=1}^{\infty} \int_{i C-t}^{i C+A-t}\left[1-F_{X}(x)\right] d x
\end{aligned}
$$

A similar result can proved for $\int_{0}^{j} h(X) d F_{X}(x)$. For proofs please refer to Appendix B.

Theorem 3.9. For $\forall j \geq 1$ and $E[x]<\infty, \int_{0}^{\infty} h(X) d F_{X}(x)=$ $\sum_{i=1}^{\infty} \int_{i C-t}^{i C+A-t}\left[1-F_{X}(x)\right] d x$ 


\subsubsection{The final expression of total observation time}

Recall from (3) that

$E[O]=\int_{t=0}^{A} E[O \mid L=t] d F_{L}(t)+\int_{t=A}^{C} E[O \mid L=t] d F_{L}(t)$.

By replacing the inner integrals by the expressions obtained in Theorems 3.8 and 3.9, we obtain

$$
\begin{gathered}
E[O]=\int_{0}^{A} \int_{0}^{\infty} g(X) d F_{X}(x) d F_{L}(t)+ \\
\int_{A}^{C} \int_{0}^{\infty} h(X) d F_{X}(x) d F_{L}(t)= \\
\int_{0}^{A}\left(\int_{0}^{A-t}\left[1-F_{X}(x)\right] d x+\sum_{i=1}^{\infty} \int_{i C-t}^{i C+A-t}\left[1-F_{X}(x)\right] d x\right) d F_{L}(t) \\
+\int_{A}^{C}\left(\sum_{i=1}^{\infty} \int_{i C-t}^{i C+A-t}\left[1-F_{X}(x)\right] d x\right) d F_{L}(t) \\
\quad E[O]=\int_{0}^{A}\left(\int_{0}^{A-t}\left[1-F_{X}(x)\right] d x\right) d F_{L}(t)+ \\
\int_{0}^{C}\left(\sum_{i=1}^{\infty} \int_{i C-t}^{i C+A-t}\left[1-F_{X}(x)\right] d x\right) d F_{L}(t)
\end{gathered}
$$

This completes the proof of Theorem 3.1.

\subsubsection{Sensitivity analysis of expected observation time}

In this section, we investigate the effect of $A$, the duration of the awake period, on $E[O]$. We define $A^{\prime}=A+h$, where $t \in\left[0, A^{\prime}\right],\left[A^{\prime}, C\right]$. Then $\varphi(i)$ is the observation time for the awake period $i$.

Next, if the event duration, $X$, is exponential, i.e. $1-$ $F_{X}(x)=e^{-\lambda x}$, then

$$
\varphi\left(A^{\prime}\right)-\varphi(A)=\frac{e^{-A \lambda}}{\lambda^{2} C}\left[1-e^{-\lambda h}\right] \cdot\left[e^{A \lambda}-1+\left(e^{-C \lambda}-1\right)^{2}\right]
$$

For the proof, please refer to Appendix B. Thus,

$$
\lim _{h \rightarrow 0} \frac{\varphi\left(A^{\prime}\right)-\varphi(A)}{h}=\frac{e^{-A \lambda}}{\lambda C} \cdot\left[e^{A \lambda}-1+\left(e^{-C \lambda}-1\right)^{2}\right]
$$

that is a constant. This means that the rate of the improvement in the observation time by increasing $A$ depends on the values of $C, A$ and $\lambda$.

\subsection{Guidelines for selecting $A$ and $C$}

In some situations (e.g. uncoordinated management among nodes, various configuration of nodes based on their deployment constraints/requirements, heterogeneity of nodes with various capabilities, different observation schedules and/or security), the sleep and awake periods are necessarily dynamic.

We now generalize the observation time for scenarios where sleep and awake periods follow a random distribution. The idea is based on external versus local view to event's arrival and can be modeled by the classic Poisson Arrival See Time Averages (PASTA), as shown by AbdelSalam and Olariu [1]. The PASTA property guarantees that the busy percentage for an external observer matches the local view. In observing the events from the viewpoint of a sensor that has sleep and awake periods, AbdelSalam and Olariu [1] showed, using a simple renewal theory argument, that the probability of finding a sensor in the awake state is

$$
\operatorname{Pr}[\{\text { sensor is awake }\}]=\frac{E[A]}{E[A]+E[S]}
$$

Due to this result, it can be assumed that our framework, i.e. Theorem 3.1, will be applicable in scenarios where sensors follow a random variable model for their sleeping-awake schedule. To summarize, we have the following.

Claim 3.10. The expected observation when random variables $A$ and $S$ are used for awake and sleep periods, equals the expected observation based on the expected value of sleep and awake random variables, i.e. $E[A]$ and $E[S]$.

\subsection{How to increase observation time}

\subsubsection{Adaptive duty cycles}

We describe situations where a different observation schedule might be required. For example, there could be two thresholds on the amount of observation, $T h_{1}$ and $T h_{2}$. Consider sleep and awake periods $S_{1}$ and $A_{1}$, respectively. When an event is observed and the amount of observation exceeds $T h_{1}$, the schedule for sleep and awake times are changed to $S_{2}$ and $A_{2}$, respectively. This can happen for many reasons. For example, after $T h_{1}$, the probability of the event happening is increased and the event should be detected faster. In this situation, for instance, $A_{2}$ is selected to be larger than $A_{1}$, and $S_{2}$ is set equal to $S_{1}$. Similarly, there can be $A_{3}$ and $S_{3}$ after $T h_{2}$. We call this an operational mode change. For example, $A_{1}$ and $S_{1}$ can be normal mode, then we switch to exceptional mode with $A_{2}$ and $S_{2}$, and later to $A_{3}$ and $S_{3}$ as critical mode.

\subsubsection{Data aggregation}

Data aggregation can be used to reduce network traffic, lessen energy consumption, boost detection accuracy, and infer new knowledge that could not be extracted from a single piece of information $[2,9,7]$. In the context of missionoriented networks, all of these are important. However, our framework focuses on information accuracy since we are quantifying the confidence of event detection, i.e. QED. We aggregate information to achieve a higher accuracy and certainty about the occurrence of an event. When an event is observed by several sensors, all observations are combined to achieve a better degree of confidence about the occurrence of the event(s) of interest. This is very helpful because we can achieve a confidence level earlier than if we had waited for one sensor to detect the event with the same level of confidence. Also, this avoids outliers, which could happen in the presence of decalibrated sensors. Our framework quantifies the total observation time and the confidence of detection by a generic sensor. Taking into account the semantics of the mission at hand, it is possible, at least in principle, to define an efficient aggregation method that meets the requirements of the mission. However, this topic is out of the scope of this paper and is being dealt with elsewhere.

\subsection{Definition of QED}

We quantify the QED as a monotonically increasing function of the observation time. Without loss of generality, a map function can transfer the observation time into the domain of $\mathbb{R}[0,1]$. Linear, exponential, and S-shaped functions are examples of map functions. The framework introduced 
in Section 3.1 shows that the observation time that is captured by each sensor is dependent on the sleeping-awake periods. The longer the awake time, the more the observations, and therefore the more certainty about the occurrence of event. We denote the map function as $\phi$. Thus,

Definition 3.11.

$$
Q E D=\phi(O)
$$

\section{SIMULATION RESULTS}

In this section, we present results of simulation in MATLAB and compare them with the described theoretical framework. Simulations are based on general time slot units, so there is no specific time unit (e.g. seconds, minutes) for the sleeping-awake schedules or event duration.

\subsection{Observation time}

In this section, we show simulation results that confirm Theorem 3.1 for the expected value of observation time. Figure 4 compares the observation between theory and simulation with constants $S=7, A=3$, and exponential event duration with parameter $\lambda=20$. It can be observed that the simulation value for expected observation time is close to its theory equivalent.

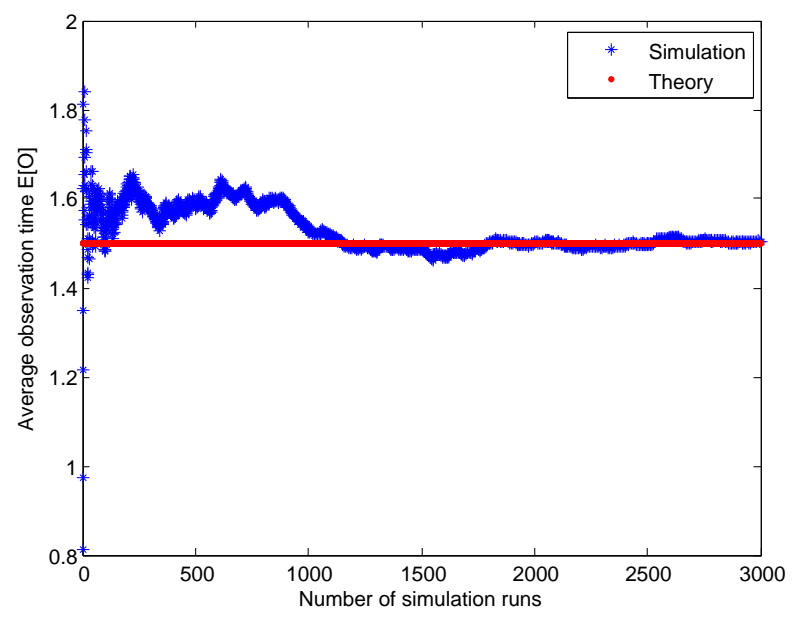

Figure 4: Expected Observation Time

The effect of a change in $A$ for a fixed cycle $C=10$ is illustrated in Figure 5. A linear growth in observation time is viewed. This confirms the sensitivity analysis in Section 3.1.6, where growth is a constant.

Table 1 shows the expected observation time value for simulation and theory for values of $C=10$ and $A=3$ when different durations for events are considered. Next, some map functions are applied to expected observation time to compute the QED on each of the rows.

\subsection{Random duty cycle}

We evaluate the effect of having random variables for sleep-awake cycles on observation time. Figure 6 shows the observation time when a fixed duty cycle is used versus a duty cycle that follows a random process. A uniform distribution is used for the selection of random variables. $A$ and $S$ will choose their values in the range $[a, b]$ and $[c, d]$, respectively. The fixed schedule will have $\frac{a+b}{2}$ and $\frac{c+d}{2}$, which

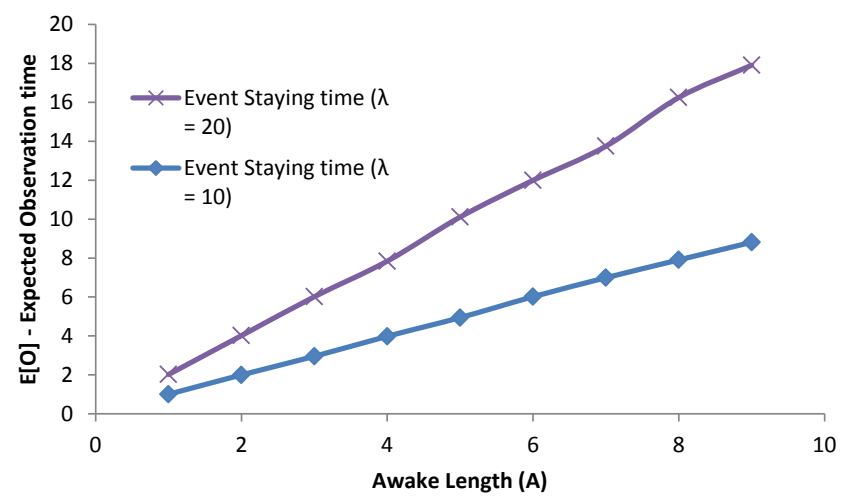

Figure 5: Expected Observation Time for various A for two different durations and $C=10$

Table 1: Expected observation time

\begin{tabular}{|c|c|c|c|c|}
\hline \multirow[t]{2}{*}{$X$ model } & \multirow[t]{2}{*}{$E[O]$} & \multicolumn{3}{|c|}{ Map Function (QED) } \\
\hline & & $\begin{array}{l}\text { S- } \\
\text { shaped }\end{array}$ & $\begin{array}{l}\text { delayed } \\
\text { step (D> } \\
0.5)\end{array}$ & $\begin{array}{l}\text { exp. } \quad(\lambda= \\
5)\end{array}$ \\
\hline $\begin{array}{l}\exp . \\
0.5)\end{array} \quad(\lambda=$ & 0.15 & 0.0069 & 0 & 0.63 \\
\hline $\begin{array}{l}\exp . \quad(\lambda= \\
1)\end{array}$ & 0.30 & 0.0071 & 0 & 0.78 \\
\hline $\begin{array}{l}\text { exp. } \quad(\lambda= \\
20)\end{array}$ & 6.00 & 0.0218 & 1 & 0.99 \\
\hline $\begin{array}{l}\text { Pareto } \\
(\alpha=2, \\
\beta=1)\end{array}$ & 0.5 & 0.0074 & 1 & 0.9 \\
\hline $\begin{array}{l}\text { Pareto } \\
(\alpha=2, \\
\beta=0.5)\end{array}$ & 0.29 & 0.0071 & 0 & 0.77 \\
\hline $\begin{array}{l}\text { Pareto } \\
(\alpha=3, \\
\beta=0.5)\end{array}$ & 0.21 & 0.0070 & 0 & 0.66 \\
\hline
\end{tabular}

correspond to $E[A]$ and $E[S]$, respectively. The x-axis shows the ratio $\frac{E[A]}{E[A]+E[S]}$. We assume $S \in[1,20]$ and $A \in[1 . . v a]$, where $v a$ is varied from 1 to 20 . $E[O]$ for each fixed and variable scenario are shown in Figure 6. The results for exponential event duration time with parameter 10 and 20, show that the expected total observation times are very close, i.e. the difference is negligible. This confirms Claim 3.10.

\section{RELATED WORK}

In event monitoring, it is key to obtain accurate information in a timely fashion while also saving energy. Satisfying these requirements while simultaneously considering the stochastic nature of an event's occurrence, has been a topic of recent interest $[11,4,5]$. For example, [5] and [11] try to design optimum strategies to cover event monitoring for an area while using minimum energy. Using mobile nodes for 


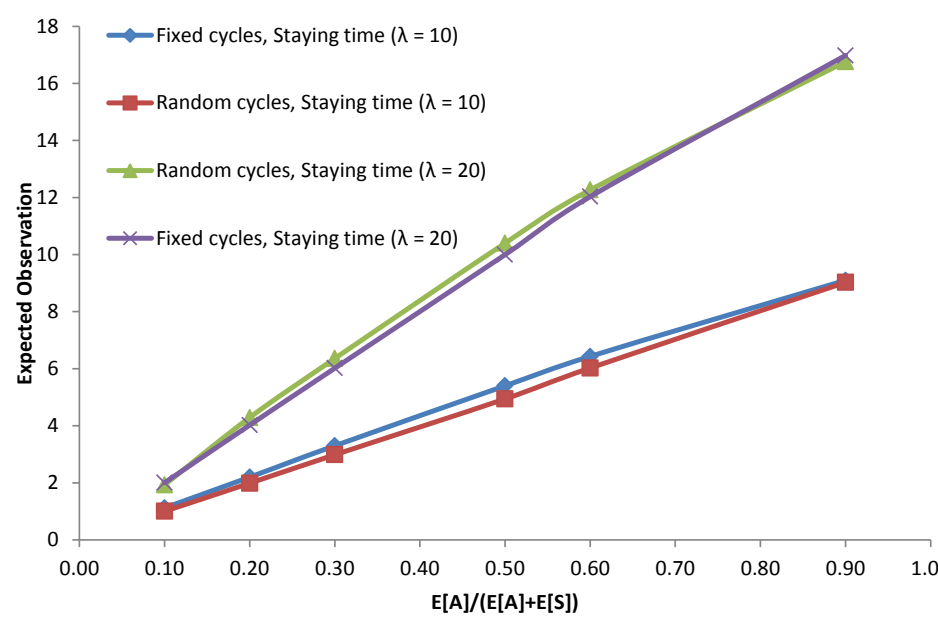

Figure 6: Comparing random and fixed duty cycles

collecting data to reduce the energy consumption has also been proposed [11, 4].

In terms of certainty and accuracy of data, even though many metrics have been defined and evaluated such as Quality of Monitoring (QoM) or Quality of Information (QoI) [10], existing literature mainly does not characterize the metric in a way that could be used simply by decision makers. Among all metrics, uncertainty of gathered information is one of the most challenging metrics due to stochastic characteristics of events, which has not been studied comprehensively. To the best of our knowledge, the only work about uncertainty of information, i.e. here QED, in sensor networks are [3] and [6]. Hossain et al. [3] characterize uncertainty based on the context, such as environmental geometry, sensor placement, orientation, and time. However, they have not looked at the stochastic occurrence of events. Work by Kessel [6] focuses on the relationship between certainty of information among nodes. So, it still suffers from not considering the stochastic occurrence of events. Furthermore, we investigate the problem with dynamic sleep/awake periods, which has not been studied before.

\section{CONCLUDING REMARKS}

In this paper we developed a framework to assess the quality of event detection (QED) in sensor networks. QED is an important design parameter particularly in mission-oriented applications because accurate information about an event must be obtained in a timely fashion. Our results show that QED is related to stochastic characteristics of problem, particularly event arrival and duration distributions as well as sensor awake and sleep period distributions. Our framework can be used to guide the design of sleep and awake periods to suit the specific needs of mission-oriented applications in sensor networks.

\section{REFERENCES}

[1] H. AbdelSalam and S. Olariu. Toward adaptive sleep schedules for balancing energy consumption in wireless sensor networks. Computers, IEEE Transactions on, 61(10):1443 -1458, Oct. 2012.
[2] I. Akyildiz, W. Su, Y. Sankarasubramaniam, and E. Cayirci. A survey on sensor networks. Communications Magazine, IEEE, 40(8):102 - 114, Aug. 2002.

[3] M. Anwar Hossain, P. Atrey, and A. El Saddik. Context-aware QoI computation in multi-sensor systems. In Mobile Ad Hoc and Sensor Systems, 2008. MASS 2008. 5th IEEE International Conference on, pages $736-741$, Oct. 2008.

[4] N. Bisnik, A. Abouzeid, and V. Isler. Stochastic event capture using mobile sensors subject to a quality metric. In Proceedings of the 12th annual international conference on Mobile computing and networking, MobiCom '06, pages 98-109, 2006.

[5] G. He and J. Hou. Tracking targets with quality in wireless sensor networks. In Network Protocols, 2005. ICNP 2005. 13th IEEE International Conference on, pages 12-74, Nov. 2005.

[6] R. Kessel. The dynamics of information fusion: Synthesis versus misassociation. In Information Fusion, 2006 9th International Conference on, pages 1 -6, July 2006.

[7] R. Kulkarni, A. Forster, and G. Venayagamoorthy. Computational intelligence in wireless sensor networks: A survey. Communications Surveys Tutorials, IEEE, 13(1):68 -96, quarter 2011.

[8] L. Oliveira and J. Rodrigues. Wireless sensor networks: a survey on environmental monitoring. Journal of Communications, 6(2), 2011.

[9] R. Rajagopalan and P. K. Varshney. Data aggregation techniques in sensor networks: A survey. Comm. Surveys \& Tutorials, IEEE, 8:48-63, 2006.

[10] V. Sachidananda, A. Khelil, and N. Suri. Quality of information in wireless sensor networks: A survey. In Proceedings of the International Conference on Information Quality, 2010.

[11] D. K. Y. Yau, N. K. Yip, C. Y. T. Ma, N. S. V. Rao, and M. Shankar. Quality of monitoring of stochastic events by periodic and proportional-share scheduling of sensor coverage. ACM Trans. Sen. Netw., 7(2):18:1-18:49, Sept. 2010.

\section{APPENDIX}

\section{A. TWO CLAIMS}

First, we prove two claims that will be used for proofs of other theorems.

Assume $X$ is a random variable and $F_{X}(x)$ is its Cumulative Distribution Function. Let $0 \leq a \leq b, \Delta(a, b)=$ $b F_{X}(b)-a F_{X}(a)$.

Claim A.1. $\Delta(a, b)=\int_{a}^{b} x d F_{X}(x)-\int_{a}^{b} F_{X}(x) d x$.

Proof. Let $u=x, v=F_{X}(x)$, according to the integration by parts theorem

$\int u d v=\int u v^{\prime} d x=u v-\int u^{\prime} v d x=x F_{X}(x)-\int F_{X}(x) d x$

we get Claim A.1 by directly integrating.

Claim A.2. If $X$ only takes positive values and has finite expectation, then $\lim _{x \rightarrow \infty} x\left[1-F_{X}(x)\right]=0$. 
Proof. Observe that $\forall x \geq 0$

$$
x\left[1-F_{X}(x)\right]=x \int_{x}^{\infty} d F_{X}(x)<\int_{x}^{\infty} u d F_{X}(u)
$$

On the other hand, since $X$ has finite expectation,

$$
\begin{gathered}
E[x]=\int_{0}^{\infty}|u| d F_{X}(u)<\infty \\
\lim _{x \rightarrow \infty} \int_{x}^{\infty} u d F_{X}(u)=0
\end{gathered}
$$

As a result,

$$
\begin{gathered}
0 \leq \lim _{x \rightarrow \infty} x\left[1-F_{X}(x)\right] \leq \lim _{x \rightarrow \infty} \int_{x}^{\infty} u d F_{X}(u)=0 \\
\lim _{x \rightarrow \infty} x\left[1-F_{X}(x)\right]=0
\end{gathered}
$$

\section{B. PROOF OF THEOREMS}

\section{B.1 Proof of Theorem 3.7}

Proof. We proceed by induction on $j$. To settle the basis, observe that for $j=1$ we have

$$
\begin{aligned}
& \int_{0}^{C-t} g(X) d F_{X}(x) \\
= & \int_{0}^{A-t} g(X) d F_{X}(x)+\int_{A-t}^{C-t} g(X) d F_{X}(x) \\
= & \int_{0}^{A-t} X d F_{X}(x)+\int_{A-t}^{C-t}(A-t) d F_{X}(x) \\
= & \int_{0}^{A-t} X d F_{X}(x)+(A-t)\left[F_{X}(C-t)-F_{X}(A-t)\right] \\
= & \int_{0}^{A-t} X d F_{X}(x)+(A-t) F_{X}(C-t)-(A-t) F_{X}(A-t) \\
= & \int_{0}^{A-t} X d F_{X}(x)+\left[(C-t) F_{X}(C-t)-(A-t) F_{X}(A-t)\right] \\
+ & (A-t) F_{X}(C-t)-(C-t) F_{X}(C-t) \\
= & \int_{0}^{C-t} X d F_{X}(x)-\int_{A-t}^{C-t}\left[1-F_{X}(x)\right] d x+S\left[1-F_{X}(C-t)\right] \\
= & \int_{0}^{A-t} X d F_{X}(x)+\Delta(A-t, C-t)-(S) F_{X}(C-t) \\
= & \int_{0}^{C-t} X d F_{X}(x)+\int_{A-t}^{C-t} X d F_{X}(x)+
\end{aligned}
$$

which is exactly the RHS of the statement at $j=1$.
We now consider an arbitrary $j>1$ and assume that the theorem is true for $j-1$. In other words, we assume that

$$
\begin{aligned}
& \int_{0}^{(j-1) C-t} g(X) d F_{X}(x) \\
& =\int_{0}^{(j-1) C-t} x d F_{X}(x)- \\
& \sum_{i=1}^{j-1} \int_{(i-1) C+A-t}^{i C-t}\left[1-F_{X}(x)\right] d x+ \\
& +(j-1) S\left[1-F_{X}[(j-1) C-t]\right]
\end{aligned}
$$

is true. With this in hand, we need to show that the statement holds for $j$.

For this purpose, we write

$$
\begin{aligned}
& \int_{0}^{j C-t} g(X) d F_{X}(x)= \\
& \int_{0}^{(j-1) C-t} g(X) d F_{X}(x)+ \\
& \int_{(j-1) C-t}^{(j-1) C+A-t} g(X) d F_{X}(x)+ \\
& \int_{(j-1) C+A-t}^{j C-t} g(X) d F_{X}(x)
\end{aligned}
$$

Then second integral would be

$$
\begin{aligned}
& \int_{(j-1) C-t}^{(j-1) C+A-t} g(X) d F_{X}(x) \\
& =\int_{(j-1) C-t}^{(j-1) C+A-t}[X-(j-1) S] d F_{X}(x) \\
& =\int_{(j-1) C-t}^{(j-1) C+A-t} X d F_{X}(x)- \\
& (j-1) S\left[F_{X}[(j-1) C+A-t]-F_{X}[(j-1) C-t]\right] \\
& =\int_{(j-1) C-t}^{(j-1) C+A-t} X d F_{X}(x) \\
& -(j-1)(S) F_{X}[(j-1) C+A-t]+ \\
& (j-1)(S) F_{X}[(j-1) C-t]
\end{aligned}
$$

and third integral will be

$$
\begin{aligned}
& \int_{(j-1) C+A-t}^{j C-t} g(X) d F_{X}(x) \\
& =\int_{(j-1) C+A-t}^{j C-t}(j A-t) d F_{X}(x) \\
& =(j A-t) \int_{(j-1) C+A-t}^{j C-t} d F_{X}(x) \\
& =(j A-t)\left[F_{X}(j C-t)-F_{X}[(j-1) C+A-t]\right] \\
& =(j A-t) F_{X}(j C-t)-(j A-t) F_{X}[(j-1) C+A-t]
\end{aligned}
$$

Thus, 


$$
\begin{aligned}
& \int_{0}^{j C-t} g(X) d F_{X}(x) \\
& =\int_{0}^{(j-1) C-t} X d F_{X}(x) \\
& -\sum_{i=1}^{j-1} \int_{(i-1) C+A-t}^{i C-t}\left[1-F_{X}(x)\right] d x \\
& +(j-1) S-(j-1) S F_{X}[(j-1) C-t] \\
& +\int_{(j-1) C-t}^{(j-1) C+A-t} X d F_{X}(x) \\
& -(j-1)(S) F_{X}[(j-1) C+A-t] \\
& +(j-1)(S) F_{X}[(j-1) C-t] \\
& +(j A-t) F_{X}(j C-t)-(j A-t) F_{X}[(j-1) C+A-t] \\
& =\int_{0}^{(j-1) C-t} X d F_{X}(x) \\
& -\sum_{i=1}^{j-1} \int_{(i-1) C+A-t}^{i C-t}\left[1-F_{X}(x)\right] d x \\
& +(j-1) S+(j A-t) F_{X}(j C-t) \\
& -[(j-1) C+A-t] F_{X}[(j-1) C+A-t] \\
& =\int_{0}^{(j-1) C+A-t} X d F_{X}(x) \\
& -\sum_{i=1}^{j-1} \int_{(i-1) C+A-t}^{i C-t}\left[1-F_{X}(x)\right] d x \\
& -\int_{(j-1) C+A-t}^{j C-t}\left[1-F_{X}(x)\right] d x \\
& +(j-1) S-j(S) F_{X}(j C-t) \\
& =\int_{0}^{j C-t} X d F_{X}(x) \\
& -\sum_{i=1}^{j} \int_{(i-1) C+A-t}^{i C-t}\left[1-F_{X}(x)\right] d x \\
& +j(S)\left[1-F_{X}(j C-t)\right]
\end{aligned}
$$

This confirms that the theorem is true.

\section{B.2 Proof of Theorem 3.8}

Let $X=j C-t$, we observe that

$$
\begin{aligned}
& \lim _{j \rightarrow \infty} j S\left[1-F_{X}(j C-t)\right] \\
& =S \lim _{j \rightarrow \infty} j\left[1-F_{X}(j C-t)\right] \\
& =S \lim _{x \rightarrow \infty} \frac{x+t}{C}\left[1-F_{X}(x)\right] \\
& =\frac{S t}{C} \lim _{x \rightarrow \infty}\left[1-F_{X}(x)\right]+ \\
& \frac{S}{C} \lim _{x \rightarrow \infty} x\left[1-F_{X}(x)\right]=0
\end{aligned}
$$

This result motivates taking limits as $j \rightarrow \infty$ in the theorem. First,

$$
\lim _{j \rightarrow \infty} \int_{0}^{j C-t} g(X) d F_{X}(x)=\int_{0}^{\infty} g(X) d F_{X}(x)
$$

Next,

$$
\lim _{j \rightarrow \infty} \int_{0}^{j C-t} X d F_{X}(x)=\int_{0}^{\infty} X d F_{X}(x)=E[x]
$$

And we just proved,

$$
\lim _{j \rightarrow \infty} j(S)\left[1-F_{X}(j C-t)\right]=0
$$

As a result,

$$
\begin{array}{r}
\int_{0}^{\infty} g(X) d F_{X}(x)=E[x] \\
-\lim _{j \rightarrow \infty} \sum_{i=1}^{j} \int_{(i-1) C+A-t}^{i C-t}\left[1-F_{X}(x)\right] d x
\end{array}
$$

We can write

$$
\lim _{j \rightarrow \infty} \sum_{i=1}^{j}\left[1-F_{X}(x)\right] d x=\sum_{i=1}^{\infty} \int_{(i-1) C+A-t)}^{i C-t}\left[1-F_{X}(x)\right] d x
$$

Thus,

$$
\begin{array}{r}
\int_{0}^{\infty} g(X) d F_{X}(x)=E[X]-\sum_{i=1}^{\infty} \int_{(i-1) C+A-t}^{i C-t}\left[1-F_{X}(x)\right] d x= \\
\int_{0}^{\infty}\left[1-F_{X}(x)\right] d x-\sum_{i=1}^{\infty} \int_{(i-1) C+A-t}^{i C-t}\left[1-F_{X}(x)\right] d x
\end{array}
$$

However, it is observable that

$$
\begin{array}{r}
\int_{0}^{\infty}\left[1-F_{X}(x)\right] d x=\int_{0}^{A-t}\left[1-F_{X}(x)\right] d x+ \\
\sum_{i=1}^{\infty} \int_{(i-1) C+A-t}^{i C-t}\left[1-F_{X}(x)\right] d x+\sum_{i=1}^{\infty} \int_{(i C-t)}^{i C+A-t}\left[1-F_{X}(x)\right] d x
\end{array}
$$

and so,

$$
\begin{array}{r}
\int_{0}^{\infty} g(X) d F_{X}(x)=\int_{0}^{A-t}\left[1-F_{X}(x)\right] d x+ \\
\sum_{i=1}^{\infty} \int_{(i C-t)}^{i C+A-t}\left[1-F_{X}(x)\right] d x
\end{array}
$$

\section{B.3 Proof of Theorem 3.9}

Proof. Induction on j. For $j=1$,

$$
\begin{aligned}
& \int_{0}^{C+A-t} h(X) d F_{X}(x)= \\
& \int_{0}^{C-t} h(X) d F_{X}(x)+\int_{C-t}^{C+A-t} h(X) d F_{X}(x)= \\
& \int_{C-t}^{C+A-t}(X+t-C) d F_{X}(x)=
\end{aligned}
$$


$\int_{C-t}^{C+A-t} X d F_{X}(x)-(C-t) \int_{C-t}^{C+A-t} d F_{X}(x)=$

$\int_{C-t}^{C+A-t} X d F_{X}(x)-(C-t)\left[F_{X}(C+A-t)-F_{X}(C-t)\right]=$

$\int_{C-t}^{C+A-t} X d F_{X}(x)$

$\left.-(C-t) F_{X}(C+A-t)+(C-t) F_{X}(C-t)\right]=$

$\int_{C-t}^{C+A-t} X d F_{X}(x)-$

$\left[(C+A-t) F_{X}(C+A-t)-\right.$

$\left.(C-t) F_{X}(C-t)\right]+A F_{X}(C+A-t)$

using Claim A.1

$$
\begin{aligned}
& =\int_{C-t}^{C+A-t} X d F_{X}(x)-\left[\int_{C-t}^{C+A-t} X d F_{X}(x)+\right. \\
& \left.\int_{C-t}^{C+A-t} F_{X}(x)\right]+A F_{X}(C+A-t) \\
& =-\int_{C-t}^{C+A-t} F_{X}(x) d x+A F_{X}(C+A-t) \\
& =\left[A-\int_{C-t}^{C+A-t} F_{X}(x) d x\right]-A\left[1-F_{X}(C+A-t)\right] \\
& =\int_{C-t}^{C+A-t}\left[1-F_{X}(x)\right] d x-A\left[1-F_{X}(C+A-t)\right]
\end{aligned}
$$

which match the right hand side for $j=1$.

For the induction step, let $j \geq 2$ be arbitrary and assume that for $j-1$

$$
\begin{aligned}
& \int_{0}^{(j-1) C+A-t} h(X) d F_{X}(x)= \\
& \sum_{i=1}^{j-1} \int_{(i C-t)}^{i C+A-t}\left[1-F_{X}(x)\right] d x- \\
& (j-1) A\left[1-F_{X}((j-1) C+A-t)\right]
\end{aligned}
$$

Then we need to show,

$$
\begin{aligned}
& \int_{0}^{j C+A-t} h(X) d F_{X}(x)= \\
& \sum_{i=1}^{j} \int_{(i C-t)}^{i C+A-t}\left[1-F_{X}(x)\right] d x-j A\left[1-F_{X}(j C+A-t)\right]
\end{aligned}
$$

The left hand side of equation would be

$$
\begin{aligned}
& \int_{0}^{j C+A-t} h(X) d F_{X}(x)= \\
& \int_{0}^{(j-1) C+A-t} h(X) d F_{X}(x)(1 \mathrm{H}) \\
& +\int_{(j-1) C+A-t}^{j C-t} h(X) d F_{X}(x)(2 \mathrm{H}) \\
& +\int_{j C-t}^{j C+A-t} h(X) d F_{X}(x)(3 \mathrm{H})
\end{aligned}
$$

The terms are named as $(1 \mathrm{H}),(2 \mathrm{H})$ and $(3 \mathrm{H})$.
Then, $(2 \mathrm{H})$ would be

$$
\begin{aligned}
& =\int_{(j-1) C+A-t}^{j C-t}(j-1) A \cdot d F_{X}(x)= \\
& (j-1) A\left[F_{X}(j C-t)-F_{X}((j-1) C+A-t)\right]= \\
& \left.(j-1) A \cdot F_{X}(j C-t)-(j-1) A F_{X}((j-1) C+A-t)\right]
\end{aligned}
$$

(3H) would be

$$
\begin{aligned}
& \int_{j C-t}^{j C+A-t} h(X) d F_{X}(x)= \\
& \int_{j C-t}^{j C+A-t}[X+t-(j-1) A-j C] d F_{X}(x)= \\
& \int_{j C-t}^{j C+A-t} X d F_{X}(x)+ \\
& \int_{j C-t}^{j C+A-t}-(j C-(j-1) A-t) d F_{X}(x)= \\
& \int_{j C-t}^{j C+A-t} X d F_{X}(x)- \\
& (j C-(j-1) A-t)\left[F_{X}(j C+A-t)-F_{X}(j C-t)\right]= \\
& \int_{j C-t}^{j C+A-t} X d F_{X}(x) \\
& -[(j C-(j-1) A-t)] F_{X}(j C+A-t)+ \\
& \left.[(j C-(j-1) A-t)] F_{X}(j C-t)\right]
\end{aligned}
$$

Thus, $(1 \mathrm{H})$ and $(2 \mathrm{H})$ would be

$$
\begin{aligned}
& =\sum_{i=1}^{j-1} \int_{i C-t}^{i C+A-t}\left[1-F_{X}(x)\right] d x \\
& -(j-1) A\left[1-F_{X}((j-1) C+A-t)\right]+ \\
& (j-1) A \cdot F_{X}(j C-t)- \\
& \left.(j-1) A \cdot F_{X}((j-1) C+A-t)\right]= \\
& \sum_{i=1}^{j-1} \int_{i C-t}^{i C+A-t}\left[1-F_{X}(x)\right] d x-(j-1) A+(j-1) A \cdot F_{X}(j C-t)
\end{aligned}
$$


Finally Equation 22 would be

$$
\begin{aligned}
& \int_{0}^{j C+A-t} h(X) d F_{X}(x)=\sum_{i=1}^{j-1} \int_{i C-t}^{i C+A-t}\left[1-F_{X}(x)\right] d x \\
& -(j-1) A+(j-1) A \cdot F_{X}(j C-t)+ \\
& \int_{j C-t}^{j C+A-t} X d F_{X}(x) \\
& -[(j C-(j-1) A-t)] F_{X}(j C+A-t)+ \\
& \left.[(j C-(j-1) A-t)] F_{X}(j C-t)\right]= \\
& \sum_{i=1}^{j-1} \int_{i C-t}^{i C+A-t}\left[1-F_{X}(x)\right] d x+ \\
& \int_{j C-t}^{j C+A-t} X d F_{X}(x)+ \\
& (j C-t) F_{X}(j C-t)-(j C+A-t) F_{X}(j C+A-t)+ \\
& (j A) F_{X}(j C+A-t)-(j-1) A \\
& =\sum_{i=1}^{j-1} \int_{i C-t}^{i C+A-t}\left[1-F_{X}(x)\right] d x+ \\
& \int_{j C-t}^{j C+A-t} X d F_{X}(x)- \\
& \Delta(j C-t, j C+A-t)+A-j A\left[1-F_{X}(j C+A-t)\right] \\
& =\sum_{i=1}^{j-1} \int_{i C-t}^{i C+A-t}\left[1-F_{X}(x)\right] d x-j A\left[1-F_{X}(j C+A-t)\right]
\end{aligned}
$$

which exactly matches the RHS.

Thus,

$$
\begin{aligned}
& \int_{0}^{\infty} h(X) d F_{X}(x)=\lim _{j \rightarrow \infty} \int_{0}^{j C+A-t} h(X) d F_{X}(x) \\
& =\lim _{j \rightarrow \infty} \sum_{i=1}^{j} \int_{i C-t}^{i C+A-t}\left[1-F_{X}(x)\right] d x- \\
& \left.\lim _{j \rightarrow \infty} j A\left[1-F_{X}(j C+A-t)\right]\right) \\
& =\sum_{i=1}^{\infty} \int_{i C-t}^{i C+A-t}\left[1-F_{X}(x)\right] d x- \\
& \left.\lim _{j \rightarrow \infty} j A\left[1-F_{X}(j C+A-t)\right]\right)
\end{aligned}
$$

we show that

$$
\lim _{j \rightarrow \infty} j A\left[1-F_{X}(j C+A-t)\right]=0
$$

write, $X=j C+A-t$, we observe that

$$
\begin{aligned}
& \lim _{j \rightarrow \infty} j A\left[1-F_{X}(j C+A-t)\right]= \\
& \lim _{X \rightarrow \infty} \frac{(X+t-A) A}{C}\left[1-F_{X}(x)\right]= \\
& \lim _{X \rightarrow \infty} \frac{(t-A) A}{C}\left[1-F_{X}\right](x)+ \\
& \lim _{X \rightarrow \infty} \frac{X A}{C}\left[1-F_{X}\right](x) \\
& =0+\frac{A}{C} \lim _{X \rightarrow \infty} X\left[1-F_{X}\right](x)
\end{aligned}
$$

By Claim A.2, it will be 0. Finally, we have

$$
\int_{0}^{\infty} h(X) d F_{X}(x)=\sum_{i=1}^{\infty} \int_{i C-t}^{i C+A-t}\left[1-F_{X}(x)\right] d x
$$

\section{B.4 Proof of E[O] sensitivity}

Proof.

$$
\begin{aligned}
& \varphi\left(A^{\prime}\right)-\varphi(A)=\int_{0}^{A^{\prime}} \int_{0}^{A^{\prime}-t}\left[1-F_{X}(x)\right] d F_{L}(t)+ \\
& \int_{0}^{C}\left(\sum_{i=1}^{\infty} \int_{i C-t}^{i C+A^{\prime}-t}\left[1-F_{X}(x)\right] d x\right) d F_{L}(t)- \\
& \int_{0}^{A} \int_{0}^{A-t}\left[1-F_{X}(x)\right] d F_{L}(t)+ \\
& \int_{0}^{C}\left(\sum_{i=1}^{\infty} \int_{i C-t}^{i C+A-t}\left[1-F_{X}(x)\right] d x\right) d F_{L}(t)= \\
& \int_{0}^{A} \int_{A-t}^{A+h-t}\left[1-F_{X}(x)\right] d F_{L}(t)+ \\
& \int_{0}^{C}\left(\sum_{i=1}^{\infty} \int_{i C+A-t}^{i C+A+h-t}\left[1-F_{X}(x)\right] d x\right) d F_{L}(t)
\end{aligned}
$$

For event duration with exponential distribution, i.e. $F_{X}(x)=$ $1-e^{-\lambda x}$, the first term is

$$
\begin{aligned}
& \int_{A-t}^{A+h-t}\left[1-F_{X}(x)\right]=\frac{1}{\lambda}\left[e^{-(A-t) \lambda}-\right. \\
& \left.e^{-(A-t+h) \lambda}\right]=\frac{e^{-(A-t) \lambda}}{\lambda}\left[1-e^{-\lambda h}\right]
\end{aligned}
$$

The second term would be

$$
\begin{aligned}
& \sum_{i=1}^{\infty} \int_{(i C+A-t)}^{i C+A+h-t} e^{-\lambda x} d x=\sum_{i=1}^{\infty} \frac{1}{\lambda} e^{-(i C+A-t) \lambda}\left[1-e^{-\lambda h}\right]= \\
& \frac{e^{-(A-t) \lambda}}{\lambda}\left[1-e^{-\lambda h}\right] \sum_{i=1}^{\infty} e^{-i C \lambda}=\frac{e^{-(A-t) \lambda}}{\lambda}\left[1-e^{-\lambda h}\right] \frac{e^{-C \lambda}}{\frac{1}{1-e^{-C \lambda}}}
\end{aligned}
$$

The sum of the two terms would be

$$
\begin{aligned}
& \int_{0}^{A} \frac{e^{-(A-t) \lambda}}{\lambda}\left[1-e^{-\lambda h}\right] d F_{L}(t)+ \\
& \int_{0}^{C} \frac{e^{-(A-t) \lambda}}{\lambda}\left[1-e^{-\lambda h}\right] \frac{e^{-C \lambda}}{\frac{1}{1-e^{-C \lambda}}} d F_{L}(t)
\end{aligned}
$$

If we assume a uniform arrival of event for $(0, \mathrm{C}]$, then $d F_{L}(t)=$ $\frac{d t}{C}$, we will have

$$
\begin{aligned}
& =\frac{e^{-A \lambda}}{\lambda}\left[1-e^{-\lambda h}\right] \cdot\left[\int_{0}^{A} \frac{e^{t \lambda}}{C} d t+\frac{e^{-C \lambda}}{\frac{1}{1-e^{-C \lambda}}} \cdot \int_{0}^{C} \frac{e^{t \lambda}}{C} d t\right] \\
& =\frac{e^{-A \lambda}}{\lambda}\left[1-e^{-\lambda h}\right] \cdot\left[\frac{e^{A \lambda}-1}{C \lambda}+\frac{e^{-C \lambda}}{\frac{1}{1-e^{-C \lambda}}} \cdot \frac{e^{C \lambda}-1}{C \lambda}\right] \\
& =\frac{e^{-A \lambda}}{\lambda^{2} C}\left[1-e^{-\lambda h}\right] \cdot\left[e^{A \lambda}-1+\left(e^{-C \lambda}-1\right)^{2}\right]
\end{aligned}
$$

so

$$
\varphi\left(A^{\prime}\right)-\varphi(A)=\frac{e^{-A \lambda}}{\lambda^{2} C}\left[1-e^{-\lambda h}\right] \cdot\left[e^{A \lambda}-1+\left(e^{-C \lambda}-1\right)^{2}\right]
$$

